\title{
50-DAY OSCILLATION of the EARTH ROTATION and SOLAR ACTIVITY
}

\author{
Li Zhian Chen Li Lin Qiao \\ Dept.of Astronomy, Beijing Normal Univ. \\ 100875 \\ Beijing ,China
}

Djurovic et. al. detected 50-day oscillation of the daily Wolf numbers and the daily apparent sunspot areas. We analysed the 50-day oscillation and discovered the closer relations between the El Nino events and the 50-day oscillation of solar activity and earth rotation. To verify the relation, in the present work, we collect longer and more data series. The result confirms that the El Nino events and the 50-day oscillation of earth rotation are caused by the 50-day fluctuation of solar activity.

The data sets are composed of the daily solar flux at $2800 \mathrm{MHz}$ (SF) relative sunspot numbers (SN), length of day (LOD) and atmospheric angular momentum (AAM).

In order to study the variation of 50-day fluctuation, all series were processed with narrow band filter,then analysed by moving autoregressive process with 2 order.

Figure 1 shows that the variable curves of LOD, AAM, SF and SN are very similar and that the range of the variable periods is about from $40 \mathrm{~d}$ to $55 \mathrm{~d}$. It merits attention that the variations 50-day fluctuation of the solar activity are before that of LOD and AAM, and that the El Nino events of the equatorial eastern Pacific happened while the periods of 50-day oscillation of solar activity were reached the min i mum values in the periods of 1972, 1976, 1982 and 1987. The result shows that solar activity is perhaps an excitation that leads to the 50-day oscillation of LOD and El Nino event.

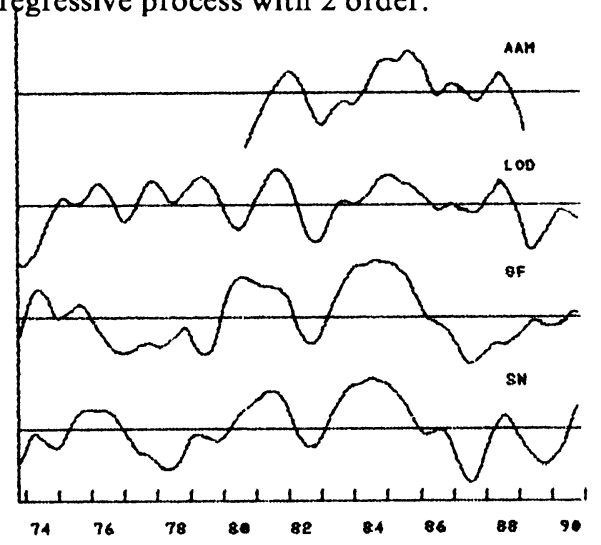

Fig.1 Variable curves of the period of 50-day oscillation 